\title{
botanika - Bremens große Entdeckerwelt Faszinierende Begegnung mit einer großen Vielfalt an Rhododendren und asiatischer Flora
}

Christiane Högermann

\section{Abstract}

The Rhododendron Park in Bremen hosts an unique collection of rhododendrons and azaleas. Attached are the botanical garden and the botanika. The botanika glass houses display the diversity of tropical and subtropical rhododendrons and the Asian flora. An interactive discovery center invites to explore the significance of biodiversity.

\section{Zusammenfassung}

Der Rhododendron-Park in Bremen beherbergt eine einzigartige Sammlung an Rhododendren und Azaleen. Angegliedert sind der Botanische Garten und die botanika. In den Schaugewächshäusern wird die Vielfalt tropischer und subtropischer Rhododendren gezeigt und die asiatische Flora thematisiert. Ein Entdeckerzentrum lädt dazu ein, auf interaktive Art und Weise die Bedeutung der biologischen Vielfalt zu erkunden.

\section{Rhododendron-Park und Botanischer Garten}

Die botanika in Bremen liegt mitten im Bremer Rhododrendron-Park. Die Hauptblütezeit der
Azaleen reicht von Januar bis März, während die meisten Rhododendren von April bis Mai blühen. Park und botanika zeigen gemeinsam viele botanische Schönheiten und seltene Vertreter der

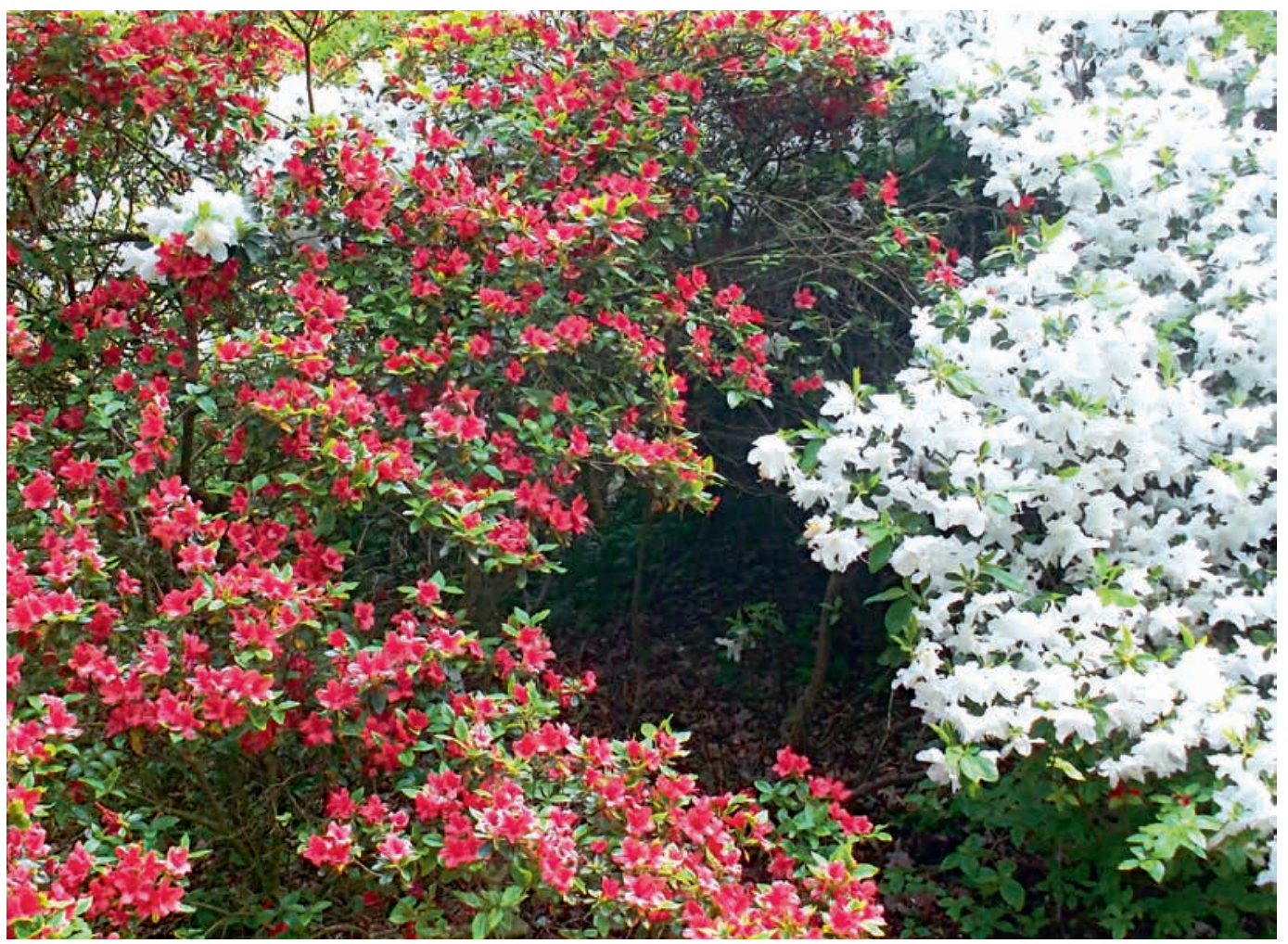

Abb. 1: Rhododendren im Freiland des Rhododendron-Parks. Links: R. 'Adonis‘, rechts: R. 'Aladdin'. (Foto: C. Högermann) 


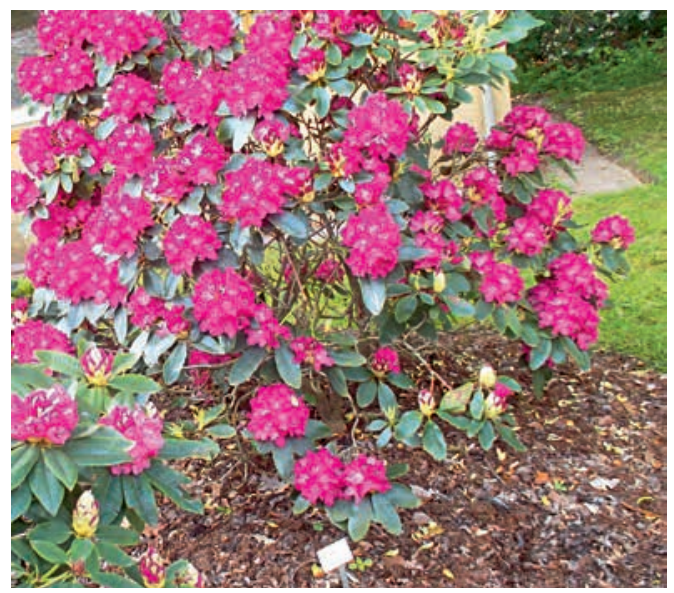

Abb. 2: Rhododendron 'Plüsch'. (Foto: C. Högermann)

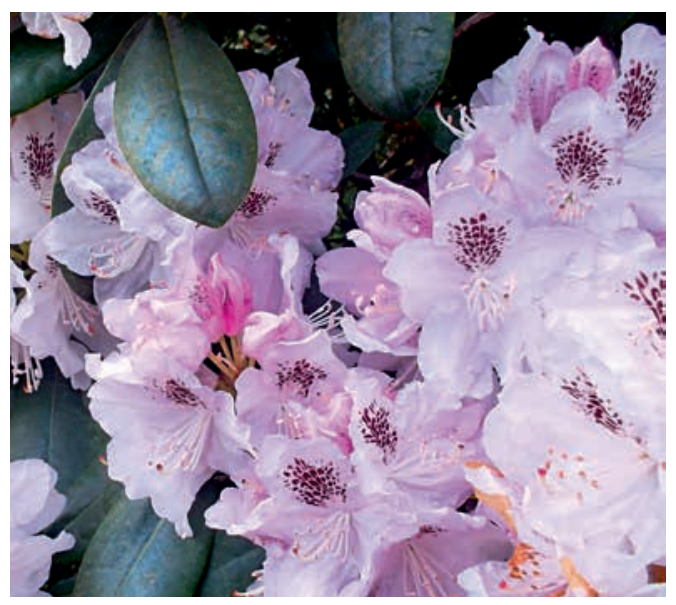

Abb. 3: Rhododendron 'Bismarck'. (Foto: C. Högermann)

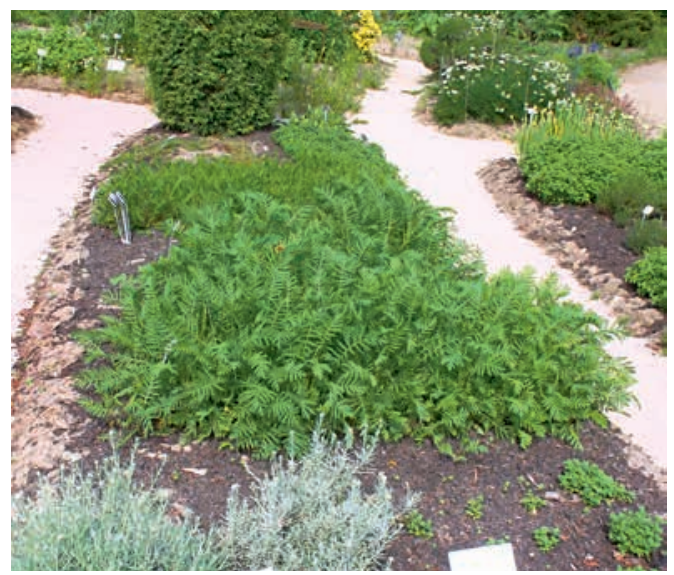

Abb. 4: Im Botanischen Garten: Heilpflanzengarten,

Abteilung für Pflanzen mit ätherischen Ölen.

(Foto: C. Högermann)

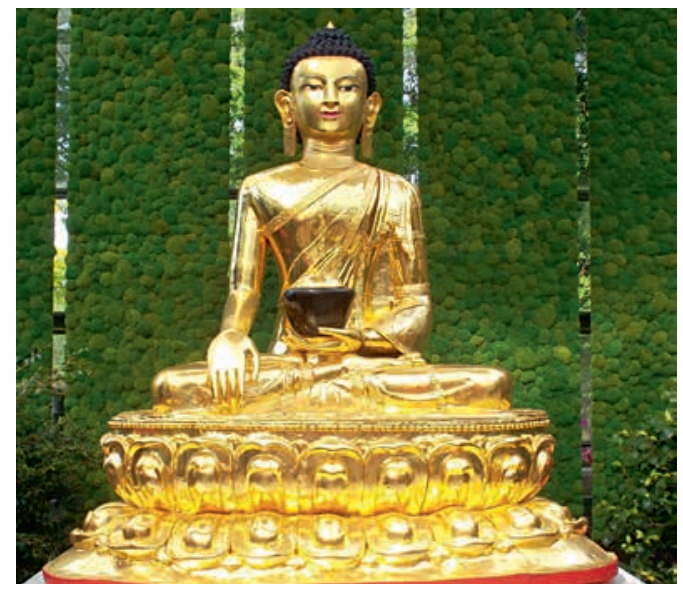

Abb. 5: Friedensbuddha für Europa. (Foto: C. Högermann)

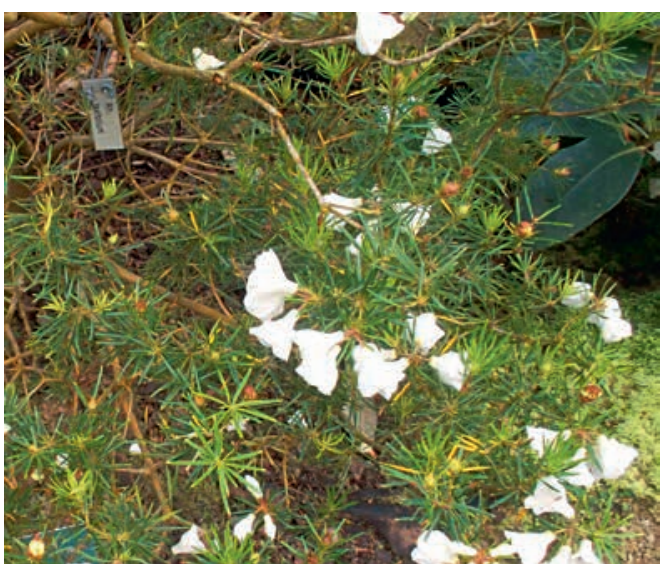

Abb. 6: Rhododendron taxifolium, eine Art, die auf der Roten Liste der bedrohten Arten steht. (Foto: C. HögermanN)

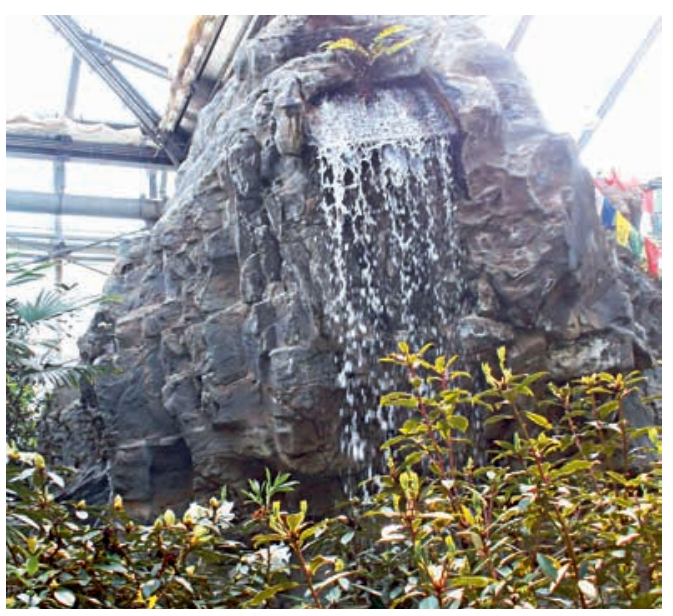

Abb. 7: Wasserfall und buddhistische Gebetsfahnen im Himalaya-Gewächshaus. (Foto: C. HögermanN) 


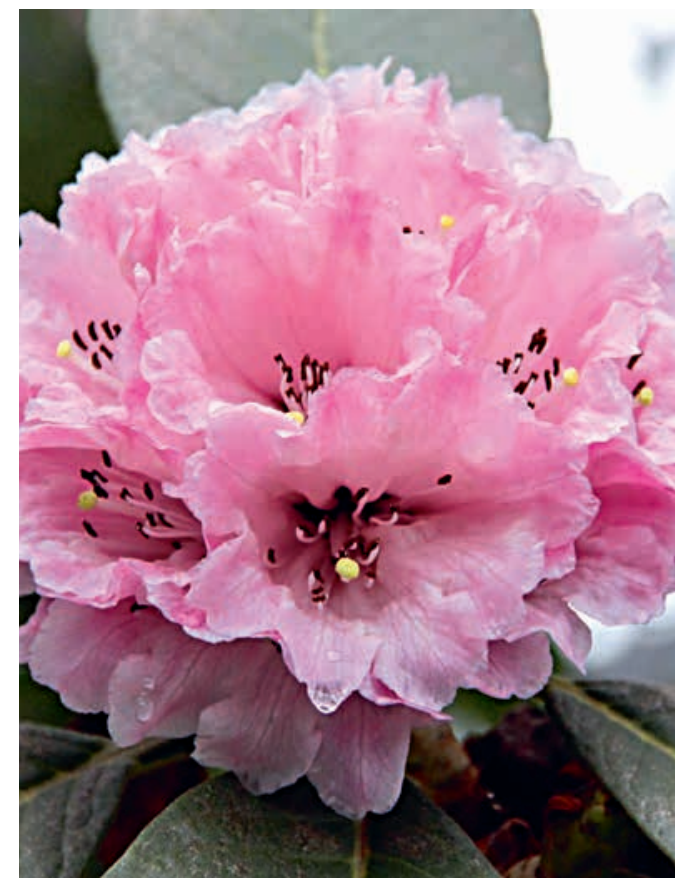

Abb. 8: Rhododendron protistum var. giganteum. (Foto: C. Högermann)

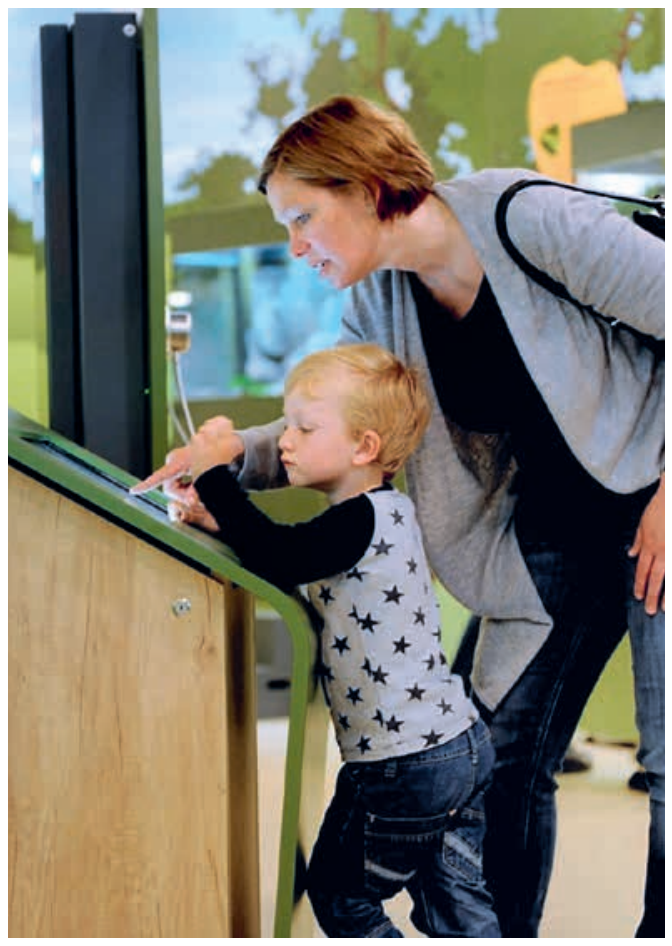

Abb. 9: Im Entdeckerzentrum. (Foto: botanika)
Azaleen und Rhododendren. Mit nahezu 600 Wildarten und über 3000 Züchtungen präsentiert Bremen eine der schönsten und größten Rhododendron-Sammlungen weltweit. Zum Bremer Rhododendron-Park gehört auch der Botanische Garten Bremen, ein traditionell nach Verwendungsmöglichkeiten von Pflanzen strukturierter botanischer Lehr- und Schaugarten. Hier gibt es z. B. die Abteilungen der nach pflanzlichen Inhaltsstoffen sortierten Heilpflanzenarten, einen Nutzpflanzenbereich und eine Sammlung homöopathisch genutzter Pflanzen.

\section{Die botanika}

Nach dem Spaziergang durch den Park geht es in die botanika. Hier erwarten die Besucherinnen und Besucher authentisch angelegte asiatische Landschaften, beginnend mit dem Japanischen Garten, wo ein Teil der Bremer Azaleensammlung ausgepflanzt ist. Besonders eindrucksvoll ist hier der europaweit einmalige 2,4 Meter hohe, vom Tibet-Haus in Neu-Delhi gestiftete Friedens-Buddha für Europa. Wie der Name schon sagt, gilt er als völker- und kulturverbindendes Friedenssymbol. Weiter geht es nach Borneo und Neuguinea. Hier erwartet die Gäste ein geheimnisvoll wirkender Ökosystemausschnitt des Regenwaldes aus dieser südostasiatischen Region mit ihren charakteristischen Rhododendren. Im anschließenden großen Himalaya-Gewächshaus begegnen wir Rhododendron giganteum. Ein chinesischer Teepavillon lädt die Besucherinnen und Besucher ein, ihre Eindrücke noch einmal Revue passieren zu lassen. Dabei kann man eine Tasse Tee genießen. Neben der pflanzlichen Vielfalt, deren Erhalt auch so etwas wie ein roter Faden der botanika ist, sind typische kulturell-rituelle Exponate wie Religionsund Fruchtbarkeitssymbole zu sehen. Botanische und kulturelle Objekte sowie Begegnungsmöglichkeiten bilden eine ansprechende Einheit, die es in dieser Form wohl sonst nirgends gibt.

Im Interaktiven Entdeckerzentrum geht es dann in eine „botanische Schule der anderen Art“: Auf 1000 Quadratmetern werden unglaubliche pflanzliche Phänomene präsentiert. Ausgeklügelte mediengestützte Techniken ermöglichen es den Besucherinnen und Besuchern, diese für sich zu er- 
schließen: Es gibt viel zu sehen, auszuprobieren, zu verstehen und zu staunen. Der Funke der Faszination pflanzlicher Angepasstheiten und arttypischer evolutiv entstandener „Errungenschaften“ springt hier garantiert über.

Bevor wir dieses von einer nahezu unglaublichen Farben-, Formen- und Artenfülle geprägte Ensemble aus botanika und Rhododendron- Park verlassen, lohnt es sich, noch einen Gang durch das Baumschulfeld mit hauseigenen Nachzuchten zu machen. Und nicht zuletzt, allerdings nicht ganzjährig, sind während mehrerer Monate im Jahr in einem Gewächshaus der botanika frei fliegende tropische Schmetterlinge zu bewundern. Weitere Informationen unter www.botanika-bremen.de sowie www.rhododendronparkbremen.de.

\section{Anschrift der Autorin}

Dr. Christiane Högermann, Blumenhaller Weg 26, 49078 Osnabrück, E-Mail: hoegermann@osnanet.de

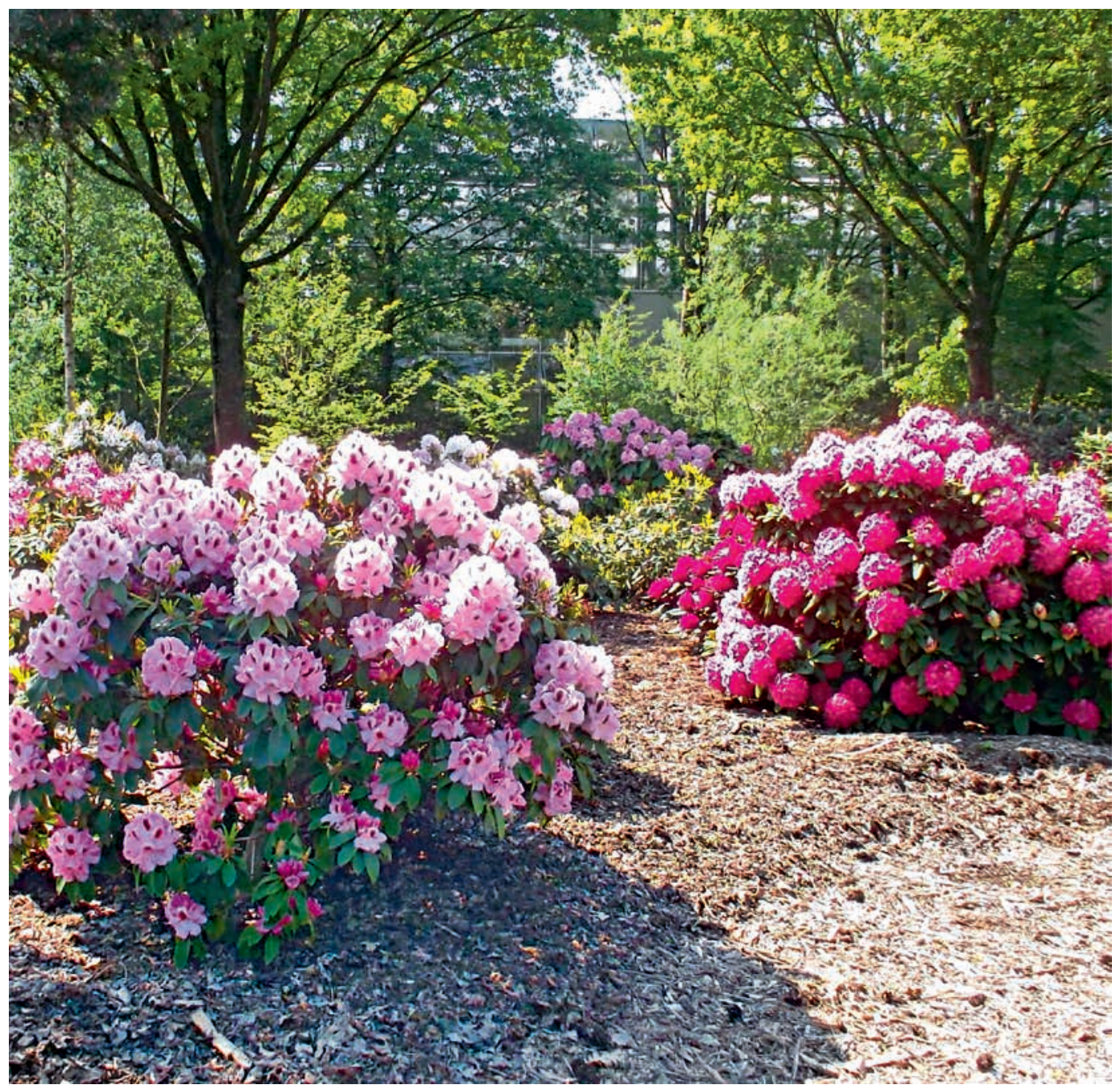

Abb. 10: Im Baumschulfeld mit Nachzuchten. (Foto: C. Högermann) 